\title{
Identifying Psychiatric Patients at Risk for Repeated Involvement in Violence: The Next Step Toward Intensive Community Treatment Programs
}

\author{
Jennifer L. Skeem, Edward P. Mulvey, Charles Lidz, William Gardner, and Carol Schubert
}

Recent studies indicate that a small, but critical subgroup of psychiatric patients is involved in a disproportionately large number of violent incidents among the mentally ill. This subgroup is an appropriate focus for intensive community-based treatment programs designed to reduce violence. However, little research has been conducted on methods for identifying patients who repeatedly become involved in violent incidents. This article describes a large follow-up study in which these patients were identified using a simple screening process that is feasible for routine use. This screening process efficiently and effectively identified a small minority of patients who were at risk for repeated involvement in violence. Patients deemed "at risk" by the screening process had an average of 7 violent incidents during a six-month follow-up period. The characteristics of these patients are described, and implications of the screening tool for conducting future research, targeting individuals for more intensive treatment services, and developing violence-focused treatment programs are discussed.

Violence tends to be concentrated in a small subgroup of the population (Blumstein, Cohen, Roth, \& Visher, 1986). This pattern applies to violence involving individuals with mental disorders as well. For example, Gardner, Mulvey, Lidz, and Shaw (1996) found that $5 \%$ of psychiatric patients followed in their community samples were involved in $45 \%$ of all reported violent incidents in the sample. More recently, Banks et al. (in press) found that $15 \%$ of psychiatric patients enrolled in their study were involved in $67 \%$ of identified violent incidents. There is some evidence that these frequently violent patients' incidents are more serious (e.g., assaults requiring medical treatment) than incidents in which "occasionally violent" patients are involved (Gardner et al., 1996).

Relatively little is known about methods for identifying patients involved in frequent and serious violence. Research on violence risk typically focuses on distinguishing patients who are likely to be involved in one or more violent incident from those with no involvement in violence (Borum, 1996). Although this work has advanced risk assessment technology (Douglas, Ogloff, Nicholson, \& Grant, 1999; Quinsey, Harris, Rice, \& Cormier, 1998; Monahan et al., 2001), most of it is nonetheless limited in its ability to guide intervention efforts with high risk patients in the community (see Skeem \& Mulvey, 2002). Differentiating individuals who are likely to commit a violent act (or multiple violent acts) from those who are not likely to commit such acts is only the first step in efficiently and effectively using treatment resources. Although this information allows for categorizing individuals into high or low risk groups, it does not go the next step in identifying those high risk individuals who pose the most predictable, ongoing risk for violence.

It is important to find and intervene with patients who are repeatedly violent for two reasons. First, identifying these patients would allow mental health

Jennifer Skeem, Department of Psychology, University of Nevada, Las Vegas. Edward Mulvey and Carol Schubert, Western Psychiatric Institute and Clinic, University of Pittsburgh School of Medicine. Charles Lidz, University of Massachusetts School of Medicine. William Gardner, University of Pittsburgh School of Medicine.

Work on this article was supported by the National Institute of Mental Health (grant \#5 R01 MH40030-12 for the collection of data, and grant \#2 T32 MH18269-14 for Dr. Skeem's postdoctoral support). We thank all the interviewers who did a diligent job of data collection, Jane Zoltun and Kenneth Conrad for their assistance with data management, and the late Saleem Shah for the initial prodding to pursue the question of changes in a patient's risk state for violence.

Correspondence concerning this article should be addressed to Jennifer L. Skeem at skeem@unlv.edu or at Department of Psychology, University of Nevada, Las Vegas, Box 455030, Las Vegas, Nevada, 89154-5030. 
systems to more efficiently allocate and use resources to reduce the incidence of violence among individuals with mental illness. Certainly, patients who are involved in even occasional acts of aggression, if those acts are serious enough, should be provided with some specialized and intensive treatment; such intervention could avert a tragedy. The most efficient use of resources, however, would be achieved by providing treatment to patients involved in both frequent and serious violence, and achievement of this goal is only possible by developing identification methods that go beyond finding people who are involved in any sort of violence.

Increased efficiency may not be simply a desirable abstract goal; it may be the sine qua non of future efforts to reduce patient violence. Dramatic changes in the organization and financing of mental healthcare in the U.S. over the last decade has led to aggressive cost containment efforts introduced by managed care companies and spending for mental health treatment declining over the past decade relative to general health care spending (U.S. Department of Health and Human Services [DHHS], 1999). Although more research is needed, this decline in spending may reflect increased barriers to access (Weissman, Pettigrew, Sotsky, \& Regier, 2000) and reliance on burdened public systems and budgets, particularly for patients in need of longer-term, intensive care (Goldman, Frank, \& McGuire, 1994; for a review, see U.S. DHHS, 1999). Increased barriers to intensive services could be particularly detrimental to efforts to intervene with patients involved in violence, since recent evidence suggests that relatively intensive mental health or substance abuse treatment may be needed to reduce violence in high risk patients. Based on a large, one-year follow-up study of a group of patients discharged from psychiatric hospitals, Monahan et al. (2001) found that patients who attended seven or more treatment sessions during a ten-week follow-up period were significantly less likely to be violent during the subsequent ten weeks than patients who had attended six or fewer sessions (even after controlling for the treatment assignment process in this observational design). Although relevant research has not yet been conducted with civil psychiatric patients, there has long been support in the correctional treatment literature for the principle that higher risk individuals require more intensive treatment services, including community-based treatment, to reduce violent recidivism (Andrews, Bonta, \& Hoge, 1990; Bonta, Wallace-Capretta, \& Rooney, 2000). Thus, prioritizing mental health resources to intensively treat the small group of patients who are frequently violent may be the only avenue left for producing a large return in terms of reducing the incidence of violence among patients.

The second reason for increasing our efforts to address patients who are frequently involved in violence is to refine methods for intervention. There is growing consensus on the need for carefully articulated violence risk reduction programs that focus on dynamic causal risk factors (Kraemer et al., 1997), that is, risk factors that are changeable and that, when changed, result in reduced violence risk (Andrews et al., 1990; Heilbrun \& Griffin, 1999; Harris \& Rice, 1994; Monahan et al., 2001; MullerIsberner \& Hodgins, 2000). Although there is evidence that correctional treatment programs that focus on criminogenic needs (e.g., entitlement, victim blaming) are effective in reducing offenders' recidivism (Andrews et al., 1990; Gendreau, 1996; Gendreau, Little \& Goggin, 1996), there apparently are no similar programs for high risk civil psychiatric patients that focus systematically and explicitly on dynamic risk factors for violence. Such treatment programs are the next logical developments.

These needed increases in efficiency of case identification and effectiveness of treatment rest on empirical progress regarding two issues. First, a method must be developed for identifying patients who are repeatedly involved in violence, not just patients who might be involved in a violent incident. This is a variation on the task undertaken in prior risk assessment research, but with the different outcome criterion of repeated involvement in violence as the prime consideration in successful case identification. Second, in order to design effective treatment, we must develop methods for determining when an individual patient is at heightened risk of being involved in violence. If patients who are repeatedly involved in violence are being treated in the community, clinicians must know what to monitor as indicators of increased risk state and what aspects of the individual's life to focus upon to reduce that risk state. The research necessary to generate information on risk state must follow patients more closely than has been done in prior research to 
capture any fluctuations in risk for violence over time, and the individual and situational correlates of these fluctuations.

In this article, we describe the initial results of a large, intensive follow-up study of patients who repeatedly become involved in violence. This larger study was designed to provide information about risk identification and risk state to inform the design of effective community-based intervention programs with patients who are repeatedly involved in violence. The results presented in this article describe our efforts to identify a group of patients for whom intensive intervention focused on violence reduction would be appropriate. Based on an actuarial model, we developed a screening process for identifying patients at risk for involvement in repeated violence. If this simple screening process effectively identifies a select group of high risk patients, it may provide a means for studying these patients and their environments and developing focused clinical interventions to reduce their involvement in violence. In the following sections, we describe the development of the screening model, present the predictions about involvement with violence that it generated, and explain the conditions under which these predictions were tested.

\section{Developing the Actuarial Prediction Model}

The larger study design required enrollment of patients who were likely to be repeatedly involved in violence for ongoing community interviewing about involvement in violence, changes in their life context (e.g., residence), and their functioning in specific realms of their life (e.g., relationships). As noted earlier, the study's screening process was not designed to discriminate between patients who would and would not be involved in violence, but instead to identify patients who were likely to become involved in repeated violence. A two-step model for identifying appropriate, repetitively violent study participants was developed, based on data from a prior study of clinical decision making with a sample of 784 patients who presented at a civil psychiatric emergency room (see Gardner et al., 1996).

The first stage of the identification process involved a review of information commonly available in medical charts (a "prescreen"). This prescreen was a regression model that included age, the number of violent incidents that occurred prior to the patient's appearance at a psychiatric emergency room (ER), and thought disorder (diagnosis of schizophrenia or current delusions, weighted negatively in the model). It identified roughly $10 \%$ of patients as eligible for the second stage of the identification process, which involved a "screening" interview and administration of the Brief Symptom Inventory (BSI; Derogatis \& Melisaratos, 1983). Specifically, eligible patients were interviewed to obtain information about the degree of drug use, the number of violent incidents that occurred during the two prior two months in the community, and scores on the BSI Hostility Scale (see Table 1). These screening variables were then combined in a regression equation to identify a final subgroup (3\%) of patients at high risk for repetitive violence in the community.

When this two-step identification process was applied to data from the prior study (see Lidz, Mulvey, \& Gardner, 1993), patients identified as likely to be involved in repeated violence had an average of 7 violent incidents during a 4-month follow-up period. Thus, the process appeared to work for its stated purpose, that is, to identify patients at risk for repeated involvement in violence. Unlike past measures, this screening process was not designed to predict involvement in a single incident of violence, and should not be used to do so. The screening process had a specificity of $99.5 \%$, but a sensitivity of only $6.9 \%$. Stated otherwise, patients who were deemed high risk by the process were very likely to be involved in violent incidents when followed up in the community in that study, but patients who were deemed low risk by the process were also involved in such incidents. This screening process was highly effective in meeting its purpose of identifying a small group of patients at high risk for frequent violence.

\section{Modifying the Prediction Model}

Making ideal systems work in real world settings often requires some adaptation. As we began using this screening process to identify participants for the intensive follow-up study, it became apparent that the empirically established (Gardner et al., 1996) 
Table 1

Empirically Established Screening Criteria (Gardner et al., 1996) versus Adapted Criteria Applied in the Follow-Up Study

\begin{tabular}{|c|c|c|}
\hline Stage of Recruitment & Empirically Established Criteria & Adapted Criteria Applied \\
\hline Prescreen & Age $=$ young $($ under 18$)$ & Age $=14-30$ years \\
\hline Prescreen & $\begin{array}{l}\text { No thought disorder } \\
\text { (schizophrenia or delusions) }\end{array}$ & $\begin{array}{l}\text { No thought disorder } \\
\text { (schizophrenia or delusions) }\end{array}$ \\
\hline Prescreen & History of violence & History of violence \\
\hline Screen & BSI hostility $\geq 8$ & BSI hostility $\geq 7$ \\
\hline Screen & $\begin{array}{l}\text { Recent heavy drug use } \\
(>\$ 5 / \text { wk of } \mathrm{MJ} \\
>1 \text { time/wk if not } \mathrm{MJ} ; \\
>1 \text { type of drug/wk) }\end{array}$ & $\begin{array}{l}\text { Recent heavy drug or alcohol use } \\
(>\$ 25 / \text { wk of MJ, } \\
\text { used drug other than } \mathrm{MJ} \\
\geq 21 \text { drinks/wk or } \geq 10 \text { drinks/day) }\end{array}$ \\
\hline Screen & Recent violence $\geq 3$ acts & Recent violence $\geq \overline{1}$ act \\
\hline
\end{tabular}

criteria would have to be modified to make them more practical for the purposes of study participant recruitment. The differences between the empirically established model and the "adapted" model used in the follow-up study are described in Table 1.

There are two substantive differences between the empirically established model and the adapted model. First, unlike the empirically established regression equation model, the adapted model weights each variable equally. This change was made because it is easier to determine whether or not a patient has each of a set of given characteristics than to make this determination, apply regression weights to each characteristic, and then sum the products. Because unit weights typically perform as well as empirically derived optimal weights (e.g., Dawes, 1979; Dawes \& Corrigan, 1974), we believed that weighing each variable equally would not substantially reduce the accuracy of the selection criteria for patients involved in repetitive violence.

Second, the adapted model modified the scope and threshold scores for some of the empirically established criteria in order to generate a large enough sample for the study. For example, with respect to scope, the drug use criterion was expanded to include the theoretically related variable of heavy alcohol use. ${ }^{1}$ Subsequent analyses of data from the prior study indicated that the vast majority of drug

\footnotetext{
${ }^{1}$ The need for this may reflect the lessening of the crack epidemic and the consequent smaller numbers of potential subjects with drug abuse problems.
}

users in the sample also used alcohol heavily, that alcohol use had a strong relationship to violence, and that few patients were exclusively drug users. Thus, the criterion for drug use was expanded to include also those patients who had heavy alcohol use, so that the overall proportion of the sample identified increased, but still stayed at about $5 \%$ of the patients who appeared at the ER. With respect to threshold scores, the BSI Hostility scale threshold was reduced from a score of 8 to 7 , and patients who had a remote history of violence but had only threatened violence within the past 2 months were included, again because an insufficient number of patients were being recruited early in the study. These alterations in the scope and threshold scores of screening criteria introduced the possibility of obtaining a sample involved in somewhat less violence, but insured that an adequate sample size would be obtained.

Third, the adapted model set thresholds for defining "young age" as 14-30 years old. Age was a continuous variable in the empirically established model. The lower limit of age 14 was set by the prior study's inclusion criteria (Lidz et al., 1993), and the upper limit of age 30 was set based on projections that less than $4 \%$ of the sample identified as high risk by the screening process would be over 30 years old.

These changes in the screening process were made to accommodate real world constraints regarding the availability of information and the characteristics of the ER sample at the time of recruitment. The changes made the screening process 
Table 2

Characteristics Associated with Patient Attrition

\begin{tabular}{|c|c|c|c|c|c|c|}
\hline \multirow[b]{3}{*}{ Characteristics } & \multicolumn{2}{|c|}{ Comparison 1} & \multicolumn{2}{|c|}{ Comparison 2} & \multicolumn{2}{|c|}{ Comparison 3} \\
\hline & \multicolumn{2}{|c|}{$\begin{array}{l}\text { Loss Between Prescreen } \\
\text { and Screening Interview }\end{array}$} & \multicolumn{2}{|c|}{$\begin{array}{c}\text { Loss Between Screening } \\
\text { Interview and Study } \\
\end{array}$} & \multicolumn{2}{|c|}{$\begin{array}{l}\text { Loss After Study } \\
\text { Enrollment }\end{array}$} \\
\hline & $\begin{array}{l}\text { Screened } \\
(n=517)\end{array}$ & $\begin{array}{l}\text { Lost to } \\
\text { Screen } \\
(n=527)\end{array}$ & $\begin{array}{l}\text { Enrolled } \\
(n=152)\end{array}$ & $\begin{array}{l}\text { Lost to } \\
\text { Study } \\
(n=19)\end{array}$ & $\begin{array}{c}\text { Completed } \\
\text { Study } \\
(n=132)\end{array}$ & $\begin{array}{l}\text { Dropped } \\
\text { from Study } \\
\quad(n=20)\end{array}$ \\
\hline Age & 20.0 & 19.9 & 21.5 & 23.5 & 21.5 & 21.5 \\
\hline Male $(\%)$ & 52.8 & $46.4^{*}$ & 48.0 & 42.1 & 47.7 & 50.0 \\
\hline White (\%) & 55.4 & 58.0 & 48.0 & 52.6 & 49.6 & 40.0 \\
\hline Education $\left(\% \geq 12^{\text {th }}\right.$ grade $)$ & 30.7 & 33.3 & 40.0 & 33.3 & 39.7 & 42.1 \\
\hline Admitted to hospital (\%) & 69.2 & $47.2 * * *$ & 70.4 & 78.9 & 68.2 & 85.0 \\
\hline Involuntary admission (\%) & 25.2 & 22.9 & 17.8 & 21.1 & 17.8 & 20.0 \\
\hline$\geq 1$ prior hospital admission $(\%)$ & 58.5 & $44.8 * * *$ & 68.9 & 66.7 & 66.9 & 63.2 \\
\hline \multicolumn{7}{|l|}{ ER Diagnosis $(\%)$} \\
\hline Psychosis (other than schizophrenia) & 8.9 & 5.9 & 7.1 & 22.2 & 5.8 & 15.0 \\
\hline Affective & 65.6 & 59.6 & 75.9 & 77.8 & 76.0 & 75.0 \\
\hline Organic & 3.7 & 3.4 & 1.4 & 0.0 & 1.7 & 0.0 \\
\hline Substance abuse & 27.9 & 23.5 & 48.2 & 66.7 & 45.5 & 65.0 \\
\hline Personality disorder & 7.7 & 6.6 & 9.2 & 15.8 & 9.1 & 15.0 \\
\hline Other & 51.6 & 57.8 & 44.8 & 36.8 & 47.9 & $20.0^{*}$ \\
\hline Axis I + substance abuse & 27.7 & $21.9 *$ & 48.2 & 66.7 & 45.5 & 65.0 \\
\hline Number of diagnoses given & 2.2 & 2.0 & 2.4 & 3.1 & 2.4 & 2.6 \\
\hline ER visit prompted by violence (\%) & 23.8 & 20.8 & 19.3 & 5.6 & 20.0 & 15.0 \\
\hline Any history of violence (\%) & 95.1 & 95.0 & 95.8 & 94.4 & 95.1 & 100.0 \\
\hline Any history of drug abuse (\%) & 66.2 & 63.6 & 87.1 & 89.5 & 87.4 & 85.0 \\
\hline Any history of alcohol abuse (\%) & 68.1 & 62.2 & 82.9 & 88.9 & 82.3 & 94.7 \\
\hline
\end{tabular}

${ }^{*} p<.05,{ }^{* * *} p<.001$

more feasible for use in routine practice. We did not believe that making these changes would greatly reduce the frequency of reports of violence in the sample that we obtained. First, these modifications were typically based on estimates of the characteristics of the $5 \%$ of the sample most frequently involved in violent incidents. Second, as suggested above, linear models of prediction are usually robust to modification in the weights (or lack thereof) applied to variables (Dawes, 1973; see also Hakeem, 1948). In fact, we believed that uncontrollable study selection issues could pose a greater threat to obtaining the desired high risk sample than the modifications to the screening criteria. For example, it is possible that high risk patients who were eligible for the study would tend to refuse to enroll in the study or fail to complete it.

The revision of the empirically established screening process described here, and its application in the intensive follow-up study provide an opportunity to examine whether the adapted screening model identifies psychiatric patients who are frequently involved in violence. If it does, researchers may be able to use this simple tool to identify a group of patients who are repeatedly involved in violence for intensive study and practitioners may be able to identify individuals who would be highly appropriate for enrollment in programs aimed at reducing violence. 


\section{METHOD}

This larger study's method was designed to identify and recruit a sample of patients who were likely to be involved in repeated violence, and follow them intensively in the community. The design included a two-stage screening process, a baseline interview, and a series of 26 weekly interviews with enrolled patients and collateral informants who were familiar with their activities.

\section{Recruiting Patients at Risk for Involvement in Repeated Violence}

Study participants were sampled from patients who were evaluated at the ER of an urban psychiatric hospital (Western Psychiatric Institute and Clinic [WPIC], Pittsburgh, PA). To identify patients who were eligible to participate in the study, a prescreening procedure and a screening interview were completed.

Prescreening process. Based on the prediction model developed in a prior study, the eligibility criteria for the Prescreen included (1) young age (1430 years), (2) a history of violence toward others, and (3) a lack of thought disorder (i.e., current diagnosis of schizophrenia or current report of delusions). Each weekday, a research associate (RA) reviewed the medical records of all patients between 14 and 30 years of age without a diagnosis of schizophrenia who had appeared at the WPIC ER during the past 24 hours or over the weekend, and coded the information required for the Prescreen (e.g., current delusions, past violence). To ensure that study participants understood the study materials and consent procedures, otherwise eligible patients were excluded if they had current diagnoses of moderate to profound mental retardation. In addition, patients who had previously completed the study were excluded from repeated participation.

Screening interview. Patients who were deemed eligible based on the Prescreen were approached by a research associate either on a hospital unit or in the community (depending upon whether they were admitted to WPIC), and invited to participate in the screening process. Patients who consented completed the Screening Interview, which addressed their drug and alcohol use during the week prior to their WPIC
ER visit, involvement in violence over the past 2 months, and current symptoms, as assessed by the BSI (Derogatis \& Melisaratos, 1983).

This information was used to determine whether patients met Screening eligibility criteria adapted from the actuarial prediction model, which included (1) recent heavy drug or alcohol use (defined below), (2) at least 1 recent violent act, ${ }^{2}$ and (3) a BSI Hostility scale score of 7 or higher. Otherwise eligible participants were excluded if (a) they could not be located, contacted and screened within 2 months of their Prescreen, or (b) had lived in the surrounding county for less than 3 months. The latter exclusion criterion was designed to minimize the likelihood of obtaining study participants who would move during the study.

\section{Interviewing Patients and Collaterals}

Patients who were deemed eligible based on the Screening Interview were invited to take part in the study, which consisted of a baseline interview and 26 weekly interviews. A collateral informant was chosen for each patient, based on the patient's nomination of individuals who knew the patient well and information was obtained weekly on each individual's frequency and duration of contact with, and judged closeness to, the patient. ${ }^{3}$ If a collateral informant had no contact with the patient or had no new knowledge about the patient during a given week, s/he did not complete an interview that week. When a collateral had no contact with, or new information about, a patient for 3 consecutive weeks, a new collateral who was more familiar with the patient's current activities was chosen to replace the old one. Based on application of these rules and the ability of the interviewers to engage collateral involvement, $73 \%$ of the follow-up interviews completed with patients had an accompanying collateral informant interview.

\footnotetext{
${ }^{2}$ Of patients deemed eligible based on the Screening Interview, most $(81 \%)$ had been involved in recent minor or serious violence. The remaining $9 \%$ deemed eligible for the study had Prescreen histories of violent acts, but had only threatened violence recently.

${ }^{3}$ For 15 enrolled patients, no collateral informant could be identified.
} 
For both patients and collaterals, interviews focused on characterizing the patient's life across the domains of interest based on weekly time frames. Participants were asked to provide recall data regarding the state of domains or events in the patient's life over the past 7 days. Variables that reflected the general state of the patient's behavior or situation (e.g., symptoms, relationship quality) were coded to reflect the state during the entire prior week. Other, more event-related variables (e.g., incidents of violence, changes in residence, drug and alcohol use) were coded to reflect their frequency and specific date of occurrence during the prior week.

Baseline interview. Eligible patients and collateral informants who agreed to take part in the study completed a Baseline Interview, which focused on characterizing the patient's life with respect to the following domains: living situation, employment or school status, social supports and relationship quality, substance use, symptoms (as assessed by the BSI), violence, and involvement in the mental health treatment and legal systems. For patients screened in the hospital, research associates conducted baseline interviews as close as possible to the date of hospital discharge (i.e., no more than 2 weeks before hospital discharge). For patients screened in the community, research associates completed baseline interviews approximately one week (5-9 days) after the Screening Interview. The average length of time between Screening and Baseline interviews was 18 days. ${ }^{4}$ The interview required approximately one hour to complete and participants were paid $\$ 10.00$.

Weekly interviews. Attempts were made to conduct 26 Weekly Interviews with patients and collateral informants, beginning either upon hospital discharge (for hospitalized patients) or one week after the baseline interview (for community patients). Typically, research associates interviewed patients in person in the community and interviewed collateral informants by telephone. Weekly Interviews focused on the same domains (e.g., violence, substance use) as the Baseline Interview, and was administered to reflect the week prior to the

\footnotetext{
${ }^{4}$ This period is lengthened by the inclusion of patients who were discharged from WPIC to another facility (e.g., jail, drug rehabilitation). For these patients, the baseline interview could be conducted as much as 6 months after the screening interview.
}

interview. Weekly Interviews were conducted no less than 5 days and no more than 9 days apart. Interviews required approximately one hour to complete and participants were paid $\$ 10.00$. Patients were informed that they were eligible for a lottery when they completed at least 23 of their 26 weekly interviews. Lotteries for two different groups of patients were held at the mid-point and end of the study, and involved first place prizes of $\$ 500.00$.

When two or fewer interviews were missed, research associates extended the recall period for violence in the next interview to include the time period that was missed. When five or more consecutive interviews were missed, the patient was dropped from the study and replaced by a new participant.

\section{Reconciling Conflicting Sources of Information}

While providing a complete picture, the use of multiple sources of information can also produce conflicting reports. When the conflict concerns whether a violent incident occurred, the most likely sources of error are arguably that the event is unknown to a source (collaterals) or a source does not wish to acknowledge the event (patients or collaterals). Therefore, any report of the occurrence of a violent incident was assumed to be a correct report. When the conflicts were about the details of a violent incident (e.g., the identity of a cocombatant), a system relying on group consensus was used to devise a "most plausible account" of the incident. This involved the principal investigators, the project coordinator, and a research associate applying a body of rules to review cases in which the details of incidents differed among the sources, and reaching an agreed upon a version of the type and timing of the incident. The manual with the coding rules governing this decision process is available from the authors.

\section{Measures}

The study measures used for this article assessed violence (from the Screening, Baseline, and Weekly Interviews), substance use (from the Screening 
Interview), and symptoms (from the Screening Interview).

Violence. The severity, frequency, and nature of patient's involvement in violent incidents for the time period defined by each interview (past 2 months for the Screening Interview; past week, otherwise) were assessed. First, patients and collateral informants were asked whether the patient had engaged in any of nine categories of aggressive acts (e.g., pushing, hitting, using a weapon) during the defined period, based on Lidz et al.'s (1993) adaptation of the Conflict Tactics Scale (CTS; Straus \& Gelles, 1990). For each category of behavior endorsed, respondents were asked to list the number of times that the act occurred. An account of each incident was then elicited, and only the most serious aggressive act for each discrete violent incident was coded. Finally, specific contextual information about each violent incident was gathered (e.g., location, co-combatant, degree of injury).

In keeping with Steadman et al. (1998), the seriousness of violence in this study was coded into two levels. Serious violence was defined as an aggressive act that resulted in physical injury (from bruises to death), a sexual assault, a threat made with a weapon in hand, or an aggressive act that involved the use of a weapon. Minor Violence was defined as physical battery that did not result in injury.

Symptoms and diagnoses. As part of each interview, patients completed the BSI (Derogatis \& Melisaratos, 1983), a self-report inventory in which patients rate how distressed they have been during the past week by each of 53 symptoms and problems, using a 5-point scale that ranges from 0 (not at all) to 4 (extremely). The BSI has well-established reliability and validity (see Derogatis, 1993 for a review). The actuarial violence prediction model (Gardner et al., 1996) and screening criteria for this study included the Hostility scale of the BSI, which consists of 5 items.

All diagnoses (Axis I and Axis II) assigned to patients, as well as current Global Assessment of Functioning (GAF; Axis IV) scores, were recorded from WPIC ER evaluations. As noted above, patients with diagnoses of schizophrenia were excluded from the study. Thus, ER diagnoses were coded into five categories, including (1) psychotic disorders other than schizophrenia (e.g., schizoaffective), ${ }^{5}$ (2) affective disorder, (3) substance abuse, (4) personality disorder, and (5) "other." When patients had both a substance abuse and other Axis I disorder, they were coded as "comorbid Axis I/substance abuse." The total number of diagnoses assigned to each patient was also calculated.

Substance use. In the Screening Interview, information about substance use was gathered by asking patients about the frequency, type, and amount of alcohol and drugs that they had used, based on the Drug and Alcohol Use Inventory developed in prior work (Lidz et al., 1993; Monahan et al., 2001). To characterize alcohol use, patients were questioned about the amount and type of alcohol they had consumed during the seven days prior to the interview (i.e., how many times they drank in the past week, and, for each day of the week on which they drank, how much beer, wine and liquor they consumed). If they reported that the week was atypical of their drinking behavior, they were questioned in the same way about their typical consumption of alcohol per week during the preceding two-month interval. Their typical rate of alcohol consumption during the follow-up interval was expressed in number of drinks per week. They were also asked about binge drinking, that is, whether they consumed more than 10 drinks at one time during the past two months. To characterize drug use, respondents were questioned about whether they used various classes of drugs during the seven days prior to the interview. If they reported that the week was atypical of their drug use, they were questioned in the same way about their typical use of drugs during the preceding 2-month interval. If they used marijuana, they were asked how much money they typically spent on it per week.

For the Screening Interview, heavy substance use over the past 2 months was defined as (a) consuming 21 or more drinks per typical week or 10 or more drinks at one time, or (b) using more than $\$ 25$-worth of marijuana per typical week or using a drug other than marijuana.

\footnotetext{
${ }^{5}$ As explained above, patients with diagnoses of schizophrenia or with current delusions were excluded from the sample, based on the Prescreen selection criteria.
} 


\section{RESULTS}

Because this project focused on the effectiveness of a screening process for identifying patients at risk for repeated involvement in violence, it was critical to analyze the study's sample selection process to determine (a) the extent to which patients who enrolled in the study represented the desired population of at-risk patients, and (b) how patients who met eligibility criteria for enrollment in the study differed in both expected and unexpected ways from those who did not. This section addresses these issues and then describes the characteristics of the enrolled sample and their rate of violence during the followup period.

\section{Subject Enrollment \& Retention}

During the data collection period (1997 through 2000 ), approximately 17,739 evaluations were completed in the WPIC ER. Based on these evaluations, 3,356 patients (between ages 14-30 with no diagnosis of schizophrenia) were prescreened (see Figure 1). Participants in this study could be lost at three successive points: (1) patients deemed eligible or potentially eligible (i.e., eligibility could not be determined based on the medical record) by the Prescreen could fail to complete a Screening Interview, (2) patients deemed eligible by the Screening Interview could fail to enroll in the study,

Figure 1

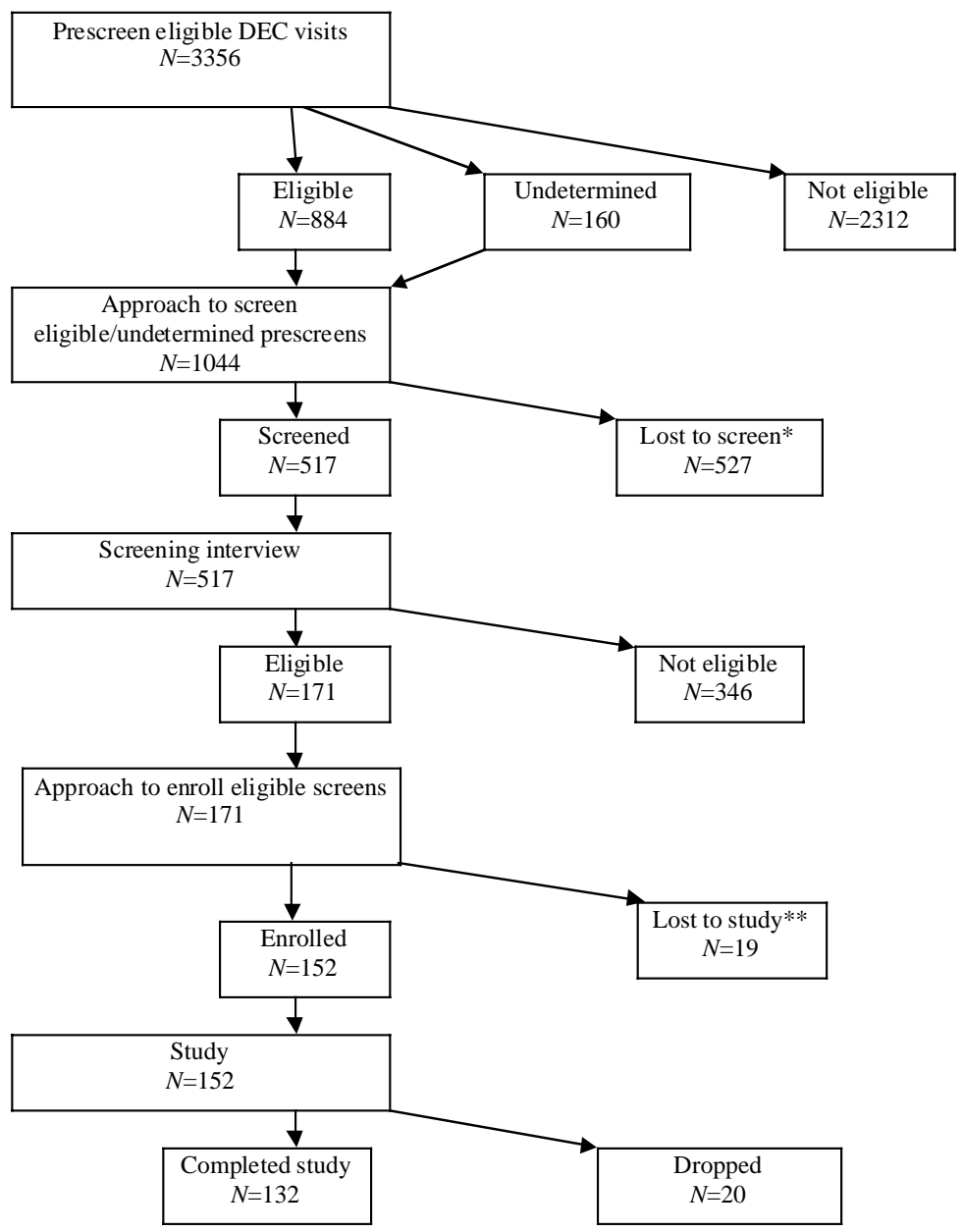

* Includes cases not found $(N=322)$ and refused $(N=205) ; * *$ Includes cases not found $(N=17)$ and refused $(N=2)$ 
and (3) enrolled patients could discontinue the study, or be dropped from the study when they missed 6 consecutive interviews. Given the nature of the study, we were most concerned about selectively losing prospective participants who were likely to be repetitively involved in violent incidents.

Most potential participants were lost at the first of these three stages: $50 \%(N=527)$ of patients approached for a Screening Interview $(N=1,044)$ either could not be found within 2 months of their Prescreen $(N=322)$ or refused the interview $(N=$ 205). Perhaps because it was more difficult to locate patients outside the hospital, patients who completed the Screening Interview may have been more symptomatic than those who did not complete the interview. Patients who did not complete the Screening Interview were less likely to be admitted to the hospital, to have prior hospital admissions, and to have a comorbid Axis I and substance abuse diagnosis than those who completed the interview (see Table 2). In order to identify any selection effects that specifically were associated with refusal of the Screening Interview, patients who refused the interview were compared with those who completed it. Patients who refused were found to differ from those who completed the interview only in that refusers were less likely to have a comorbid Axis I and substance abuse diagnosis, $\chi^{2}(1, N=674)=6.46$, $\mathrm{p}=.011$ (refused $=28 \%$; screened $=18 \%$ ).

Once patients completed the Screening Interview, they were substantially less likely to be lost to the study. Only $11 \%(N=19)$ of screened eligible patients could not be found for a baseline interview $(N=17)$ or refused participation in the study $(N=$ 2 ). No statistically significant differences were detected between eligible patients who enrolled or failed to enroll in the study, likely based in part on limited power (for a descriptive comparison, see Table 2). Only $13 \%(N=20)$ of enrolled patients were dropped from the study because they missed 5 or more consecutive weekly interviews $(N=16)$, or chose to discontinue $(N=4)$. Patients who were dropped from the study differed significantly from those who completed the study only in that they were more likely to obtain "other" diagnoses (Table 2). Again, this limited effect may be partially attributable to low power.
To estimate whether particularly violent patients were being selectively lost from the study, available data from the weekly follow-ups on patients who discontinued the study ( $N=15$ of 20 "dropped") were compared to data on patients who completed the study ( $N=132$ "enrolled"). Specifically, the ratio of the number of violent incidents that occurred during the patient's enrollment to the number of follow-ups that the patient completed were computed for both the enrolled group $(M=.29, S D=.30)$ and the dropped group $(M=.53, S D=.47)$. The difference between groups was statistically significant, $t$ (145) $=-2.8, p<.01$. For descriptive purposes, the ratio of the number of violent incidents that occurred to the proportion of follow-ups that patients completed (a more interpretable figure) is provided here. For the enrolled patients, this was $7.5(S D=7.9)$, which was substantially lower than that of the dropped cases $(M=16.6, S D=15.6)$. This suggests that the few $(13 \%, N=20)$ patients who were lost to the study after enrollment were more often violent than those who completed the study.

\section{Characteristics Associated with Eligibility Criteria}

Unintended selection effects could be associated not only with subject attrition, but also with the study's eligibility criteria. Thus, the characteristics of patients deemed eligible versus ineligible by the Prescreen and Screening Interview were compared. As shown in Table 3, several case characteristics were associated with the Prescreen eligibility criteria, which included a history of violence. Patients deemed eligible by the Prescreen were younger, more likely to be male, less likely to be White, and less educated than those deemed ineligible. They were also more likely to be admitted to the hospital, to be admitted involuntarily, to have prior admissions, to have a substance use history, and to have substance abuse, comorbid substance abuse and Axis I, and "other" diagnoses than ineligible patients. Finally, because they were Prescreen criteria, eligible patients were more likely to have a history of violence, an arrest history, and an emergency room visit precipitated by violence than ineligible patients. 
Table 3

Characteristics Associated with Study Eligibility Criteria

\begin{tabular}{|c|c|c|c|c|}
\hline \multirow[b]{2}{*}{ Characteristics } & \multicolumn{2}{|c|}{ Prescreen Criteria } & \multicolumn{2}{|c|}{ Screen Criteria } \\
\hline & $\begin{array}{l}\text { Eligible } \\
(n=884)\end{array}$ & $\begin{array}{l}\text { Ineligible } \\
(n=2,312)\end{array}$ & $\begin{array}{l}\text { Eligible } \\
(n=171)\end{array}$ & $\begin{array}{l}\text { Ineligible } \\
(n=346)\end{array}$ \\
\hline Age $\dagger$ & 19.65 & $21.41 * * *$ & 21.70 & $19.12 * * *$ \\
\hline Male (\%) & 51.50 & $43.20 * * *$ & 47.40 & 55.50 \\
\hline White $(\%)$ & 56.50 & $74.20 * * *$ & 48.80 & $58.70 *$ \\
\hline Education $\left(\% \geq 12^{\text {th }}\right.$ grade $)$ & 28.31 & $53.94 * * *$ & 39.26 & $26.40 * *$ \\
\hline Admitted to hospital (\%) & 66.60 & $58.70 * * *$ & 71.30 & 68.20 \\
\hline Involuntary admission (\%) & 26.77 & $13.33 * * *$ & 18.45 & $28.44 *$ \\
\hline$\geq 1$ prior hospital admission $(\%)$ & 55.20 & $38.40 * * *$ & 66.40 & $54.70^{*}$ \\
\hline \multicolumn{5}{|l|}{ ER Diagnosis $(\%)$} \\
\hline Psychosis (not schizophrenia) & 8.00 & 6.71 & 8.80 & 8.95 \\
\hline Affective & 64.48 & 67.15 & 76.10 & $60.49 * *$ \\
\hline Organic & 3.52 & 3.93 & 1.25 & $4.94 *$ \\
\hline Substance abuse & 26.18 & $21.11 * *$ & 50.31 & $16.97 * * *$ \\
\hline Personality disorder & 7.27 & 6.89 & 10.69 & 6.17 \\
\hline Other & 53.82 & $48.57^{*}$ & 43.40 & $55.56^{*}$ \\
\hline Axis I + substance abuse & 25.57 & $20.16^{* *}$ & 50.31 & $16.67 * * *$ \\
\hline Number of diagnoses given & 2.13 & $1.90 * * *$ & 2.50 & $1.99 * * *$ \\
\hline ER visit prompted by violence (\%) & 25.70 & $4.40 * * *$ & 17.80 & $26.80^{*}$ \\
\hline Any history of violence $(\%) \dagger$ & 95.20 & $30.05 * * *$ & 95.63 & 94.92 \\
\hline Any history of drug abuse (\%) & 65.50 & $49.20 * * *$ & 87.30 & $55.30 * * *$ \\
\hline Any history of alcohol abuse (\%) & 66.50 & $57.30 * * *$ & 84.50 & $59.70 * * *$ \\
\hline $\begin{array}{l}\text { Number of violent and aggressive } \\
\text { incidents over past } 2 \text { months }+\end{array}$ & $\mathrm{n} / \mathrm{a}$ & $\mathrm{n} / \mathrm{a}$ & 6.97 & 4.57 \\
\hline
\end{tabular}

$* p<.05, * * p<.01, * * * p<.001$

$\dagger$ Included in Prescreen eligibility criteria

\$ Included in Screen eligibility criteria

Also shown in Table 3, there were fewer differences between patients deemed eligible versus ineligible for the study based on the Screening criteria, which included recent substance abuse and violence. Eligible patients were older, less likely to be White, and more highly educated than ineligible patients. They were also more likely to have affective, substance abuse, "other" and comorbid Axis I and substance abuse diagnoses than ineligible patients. Finally, eligible patients were more likely to have recent histories of substance use than ineligible patients.
In summary, these results suggest that the sample differs in both intended and unintended ways from the population of patients typically seen in a psychiatric emergency room. Specifically, the sample meets more planned selection criteria (e.g., violence and substance use histories), but is also more symptomatic and limited in functioning (e.g., multiple diagnoses and hospital admissions; limited education) than the general civil psychiatric population. Although the sample is also less likely to be White, race/ethnicity adds no unique variance 
to predicting eligibility status once the effect of the selection criteria are taken into account. ${ }^{6}$

\section{Sample Description}

The characteristics of the 132 patients who completed this study are described in Table 2. Patients are young $(M=21$ years, $S D=6)$ men and women (52\%) who are about equally likely to be White or African American (49\%; "Other"= 2\%). Of the 83 patients age 18 and older, $65 \%$ have attained at least a high school degree and one-third live with their parents. Patients were likely to obtain diagnoses for affective (76\%) and substance abuse (45\%) disorders as well as comorbid Axis I and substance abuse disorders (45\%). They had an average of 1.7 prior psychiatric hospitalizations $(S D$ $=2.5$ ), and $60 \%$ had a recorded history of attempted suicide.

At the time of their Screening Interview, patients who completed the study obtained an average BSI

\footnotetext{
${ }^{6}$ To determine the extent to which race/ethnicity and other variables were predictive of eligibility status after the eligibility criteria were taken into account, two hierarchical logistic regression analyses were performed. In the first analysis, relevant discriminating variables summarized in Table 3 (e.g., age, race, education) were used to predict patients' Prescreen eligibility status (eligible/ineligible) after entering the patient's history of violence and current thought disorder (current delusions/ diagnosis of schizophrenia) as covariates. These two variables were entered as covariates because they were used to select the sample and this analysis was designed to determine if any case characteristics were independently associated with case selection. There was, of course, a good model fit (discrimination among groups) on the basis of the covariates alone, $\chi^{2}(3$, $N=2,393)=908.12, p<.001 ; R^{2}=.32$. Even after taking these covariates into account, however, several variables (age, diagnosis, history of alcohol use and hospitalization, and hospital admission and legal status) contributed unique variance to predicting eligibility status, $\chi^{2}(8, N=2,393)=999.00, p<.001$, $R^{2}=.34$. Notably, these variables did not include race/ethnicity. In the second analysis, relevant discriminating variables summarized in Table 3 were used to predict patients' Screening eligibility status (eligible/ineligible) after entering indices of patient's recent violence and drug use and BSI hostility scores (the Screening criteria) as covariates. There was a very good model fit on the basis of the covariates alone, $\chi^{2}(6, N=294)=$ $163.44, p<.001 ; R^{2}=.43$. After taking these covariates into account, none of predictor variables entered the equation, suggesting that these variables did not contribute unique variance to the eligibility criteria in predicting eligibility status.
}

Global Severity Index of $2.15(S D=.85) .^{7}$ This level of general symptomatology is somewhat elevated, compared to normative data for adult psychiatric inpatients. Specifically, it is equivalent to a t-score of 63 for male inpatients and 59 for female inpatients (Derogatis, 1993). Patients' average BSI subscale scores were generally in the range expected for psychiatric inpatients, with the exception of their two highest scores, which were on the Hostility $(M=$ $3.1, S D=.74, t=66$ and 65 [men and women, respectively]) and Paranoid scales $(M=2.6, S D=$ $.99 ; t=66$ and 62 [men and women]). Because high scores on the Hostility scale were an eligibility requirement, the former result is not surprising. The Paranoid scale arguably reflects a related construct, given its focus on hostility, cynicism, and suspiciousness (e.g., "People are unfriendly").

During the 2-month period preceding the Screening Interview, patients reported consuming an average of $17(S D=29)$ drinks per typical week, and $55 \%$ of these patients had binged on alcohol at least once. Most patients $(86 \%)$ had used a street drug during this period, and $70 \%$ had engaged in "heavy" drug use, as defined above. During the same 2-month period prior to hospitalization, patients who completed the study had an average of $3.1(S D=$ $4.9)$ violent incidents, including $1.8(S D=4.4)$ minor violent incidents and $1.3(S D=1.4)$ serious violent incidents. ${ }^{8}$

Patients' descriptions of their participation in treatment at the time of the Baseline interview were used to characterize their involvement with the mental health system at the beginning of the study. Most $(93 \%)$ patients were involved in psychiatric treatment during the week preceding the baseline interview (i.e., hospitalization; partial hospitalization; medication management; individual, group, or family psychotherapy; case management; vocational rehabilitation; substance abuse treatment). Although $20 \%$ of these patients were still hospital-

\footnotetext{
${ }^{7}$ All statistics from the Screening Interview that are reported in this paragraph and the following paragraph are based on 121 of the $132(92 \%)$ patients who completed this study. For these 121 patients, we have written records of their informed consent for the Screening Interview.

${ }^{8}$ These violence statistics are based on the 121 (of 132 total) patients who completed the study because written consent was available only for these 121 patients.
} 
ized at the time of the baseline interview, $52 \%$ of patients had attended a medication management session and $49 \%$ had participated in individual psychotherapy.

Patients were less likely to be involved in treatment at the end of the study, which occurred roughly six months after their discharge from the index hospitalization. Of the 124 patients who completed the study and did not miss their final interview, only $60 \%$ were involved in any form of psychiatric treatment. During the last week of the study, the most frequently attended forms of treatment were individual psychotherapy (14\%), inpatient hospitalization (7\%), and medication management (7\%). Patients' treatment sessions typically were scheduled on less than a weekly basis, as evidenced by the fact that $36 \%$ of patients said that they had not quit individual psychotherapy and $35 \%$ reported that they had not quit taking psychiatric medications.

\section{Prevalence Rates of Patient Violence}

Prevalence rates of patient involvement in violence were estimated based on a sample of 109 $(83 \%)$ patients who completed the study and missed no more than two consecutive weekly follow-up interviews. Patients who missed no more than two consecutive interviews were selected because when two or fewer interviews were missed, the recall period for violence was extended in the next interview to include the time period that was missed. Thus, for these 109 patients, data on violence was complete.

The vast majority $(89 \%)$ of these patients were involved in at least one violent incident during the 6 month follow-up period: $84 \%$ of patients were involved at least one incident of minor violence, as defined above, and $67 \%$ were involved in at least one incident of serious violence. Moreover, most $(81 \%)$ of these patients were involved in two or more serious or minor violent incidents. Specifically, patients were involved in an average of $2.6(S D=$ 3.3) incidents of serious violence, and an average of $4.5(S D=4.8)$ incidents of minor violence during the 26-week follow-up period. They had an average of $7.2(S D=7.1)$ total violent incidents (serious + minor) during this follow-up period. ${ }^{9}$

\section{DISCUSSION}

The screening process examined in this study is designed to provide researchers and practitioners with a simple tool for identifying psychiatric patients who are repeatedly involved in violence. This screening process modified an actuarial model derived from prior data to make it more feasible to apply in real world settings. This screening process identified a sample of patients who were typically $(89 \%)$ involved in violence, and, more importantly, were frequently involved in violence. During a sixmonth follow-up period, these patients were involved in an average number of 7 violent incidents, three of which were classified as serious violence (e.g., physical battery with injury, sexual assault, use of a weapon). Thus, the screening process identifies a specific class of patients who are at risk for repeated violence in the community.

The patients identified are young, non-psychotic, and hostile (as assessed by the BSI), with heavy substance use and histories of violent behavior. These patients typically obtain diagnoses of affective disorders and, to a lesser extent, substance abuse disorders or comorbid Axis I and substance abuse disorders. ${ }^{10}$ The screening process was based on an actuarial model for predicting repeated violent incidents that was developed in a prior study. Thus, the class of patients identified by this screening process does not represent all psychiatric patients at

\footnotetext{
9 These figures slightly underestimate the number of violent incidents that occurred in this sample because they are based on a dataset in which only the most violent incident that occurred during each day of the study was recorded. When patients were involved in more than one violent incident per day, only the most serious incident was considered for these analyses.

${ }^{10}$ The extent to which these repetitively violent patients share characteristics with less frequently violent patients remains to be determined by studies that compare these two groups. Wellestablished risk factors for the occurrence or non-occurrence of violence include past violence, substance abuse, and young age (e.g., Monahan et al., 2001). Future comparative studies will help to determine whether repetitively violent patients differ from less frequently violent patients in degree or in kind.
} 
high risk for violence. Patients who do not meet the screening criteria may still be involved in violent incidents; patients who do meet screening criteria, however, are at great risk for involvement in repeated violence. Thus, the screening process clearly and relatively easily identifies a clinically important subgroup of patients who are at high risk for repeated involvement in violence. It is also worth noting that the individuals lost to the study appear to be even more regularly violent than the ones retained, highlighting the need for intensive initial outreach efforts in any program aimed at intervening with the most repeatedly violent patients.

\section{Limitations}

Because this study did not include a control group, the extent to which the patients identified as high risk by this simple screening process are more frequently violent than those who are identified as ineligible is unknown. The base rate and frequency of violence among general psychiatric patients is substantially lower than that among patients identified with this screening process. Based on a large sample of psychiatric patients who were followed for one year, Monahan et al. (2001) found that $28 \%$ of patients committed a violent act, and $12 \%$ of patients were involved in two or more violent acts. In this study, which involved a follow-up period only half as long, $67 \%$ of patients were involved in serious violence, and $49 \%$ were involved in two or more serious violent incidents. Although these differences are quite striking, a replication study is necessary to compare directly the violence rates among those who "screen in" and "screen out." A replication study also would be valuable for assessing the sensitivity and specificity of this modified screening process (see Gardner et al., 1996) and for verifying the characteristics of patients identified as frequently violent by the screening process. Many subjects in this study were lost before the screening interview could be completed. Analyses indicate that, relative to those who were lost, patients who completed this interview were more symptomatic (i.e., more likely to be hospitalized and therefore easier to locate). A replication study would help to determine whether there were differences more important than these that were not detected in this study. Nevertheless, although further studies would be informative, the results of this study independently suggest that the screening process is useful for its intended purpose, that is, for identifying a specific class of patients who are at clear risk for repeated violence in the community.

\section{Implications for Research}

This study has several implications for future research on patients who are repeatedly involved in violent incidents. Because the screening process identifies patients at risk for repeated violence, this study will enable useful research on the nature, variability, and clinical needs of this important subgroup of patients. The larger study described in this article, aimed at identifying changeable risk factors that contribute to patients' involvement in violence, is a first step in this research agenda. There is a substantial amount of research on relatively stable (e.g., arrest history, psychopathy, age) and ostensibly changeable (e.g., substance use, clinical symptomatology) risk factors for psychiatric patients' violence (for a review, see Monahan et al., 2001). However, few studies directly assess whether changes in risk factors are related to the proximate occurrence of violence (Hanson \& Harris, 1998, 2000). This study's screening process yields this critical sample based on a simple set of indicators that are feasible to collect in routine practice. If applied in other settings, it could foster the growth of the field's knowledge base about this patient subgroup and the development of treatment programs targeting changeable, causal risk factors for violence (see Kraemer et al., 1997).

Using this simple screening process to identify repetitively violent patients could also promote innovative approaches to the assessment of intervention outcomes. As argued by Henry (1996), efforts to empirically support interventions are "a function of the congruence among how patient problems, therapeutic change processes, and clinical outcomes are conceptualized and measured" (p. 1263). Violence risk reduction interventions have a substantial advantage in achieving "problemtreatment-outcome congruence," given that the "problem" and "outcome" can be predefined simply as patient involvement in violence. For example, if 
a screening process like the one outlined here were used to identify patients, one could (a) make projections about the expected level of patient involvement in violence, given the absence of a particular intervention, and (b) use these projections to assess whether participation in the intervention substantially reduced violence below that expected value. The identification of congruent therapeutic change processes will be facilitated by increased knowledge about what causes changes in high risk patient's risk state.

\section{Implications for Practice}

As explained above, this study provides guidance for future research to (a) increase our understanding of risk state and dynamic risk factors for violence, (b) use this knowledge to design treatment programs to reduce violence risk, and (c) study the outcome of these treatment programs. This study also has immediate practical implications for administrators and clinicians who wish to identify patients at high risk for frequent violence and ensure that they receive intensive services. A chief concern in designing the study's screening process was its feasibility for identifying a small, high risk group of patients. An appealing aspect of this process is ease of use: at the prescreen level, one merely considers patients' age, diagnosis, and recent violence. The minority (less than 1/3) of patients who are young, non-thought-disordered, and have recently been violent then complete a brief symptom checklist and an interview focused on their recent substance use. In this study, this two-stage process identified approximately $5 \%$ of over 3,000 patients. In resource-poor mental health systems, such rapid, inexpensive identification of this small patient subgroup can be a valuable method for quickly focusing services.

This patient subgroup at high risk for repeated involvement in violence may, in fact, require some specialized approaches to increase their adherence to, and benefit from, treatment. Shortly after their visit to a psychiatric emergency room, most (93\%) of the high risk patients in this study were involved in some kind of mental health or substance abuse treatment. At the end of the six-month follow-up period, however, only $56 \%$ of patients were involved in outpatient treatment. This may not be too surprising because all of these high risk patients abuse substances heavily. Substance abuse disorders consistently are shown to complicate "first-line" treatments for Axis I disorders and to predict poor treatment adherence and outcome (for reviews, see Drake, Mercer-McFadden, Mueser, McHugo, \& Bond, 1998; Miller \& Rollnick, 1991). However, recent research suggests that "dual diagnosis" programs that integrate mental health and substance abuse treatment and focus on enhancing motivation rather than "forcing" and "confronting" can improve treatment adherence and outcome for these patients (see Daley \& Zuckoff, 1998; Drake et al., 1998; Martino, Carroll, O’Malley, \& Rounsaville, 2000; Swanson, Pantalon, \& Cohen, 1999). Clearly, these approaches to treatment engagement appear relevant for patients at high risk for frequent violence.

Regardless of the intensity and nature of treatment that proves to be most effective for these patients, the screening process described here appears to identify a unique subgroup of psychiatric patients who are at high risk for repeated involvement in violence. As argued above, this patient subgroup warrants further scholarly, social policy, and clinical attention. Ideally, the availability of this screening process will foster a better understanding of these patients and will ultimately lead to empirically supported interventions for reducing their violence potential and meeting their treatment needs. These developments are the necessary next steps in aiding clinicians to safely and effectively treat this difficult group of patients in the community.

\section{REFERENCES}

Andrews, D., Bonta, J., \& Hoge, R. (1990). Classification for effective rehabilitation: Rediscovering psychology. Criminal Justice and Behavior, 17, 19-52.

Banks, S., Robbins, P., Silver, E., Vesselinov, R., Steadman, H., Monahan, J., Mulvey, E., Appelbaum, P., Grisso, T., \& Roth, L. (in press). A multiple models approach to violence risk assessment among people with mental disorder. Criminal Justice and Behavior.

Borum, R. (1996). Improving the clinical practice of violence risk assessment: Technology, guidelines, and training. American Psychologist, 51, 945-956.

Blumstein, A., Cohen, J., Roth, J., \& Visher, C. (1986). Criminal careers and "career criminals." Washington, DC: National Academy Press. 
Daley, D., \& Zuckoff, A. (1998). Improving compliance with the initial outpatient session among discharged inpatient dual diagnosis clients. Social Work, 43, 470-473.

Dawes, R. (1979). The robust beauty of improper linear models in decision making. American Psychologist, 34, 571-582.

Dawes, R., \& Corrigan, B. (1974). Linear models in decision making. Psychological Bulletin, 81, 95-106.

Derogatis, L. (1993). Brief Symptom Inventory: Administration, scoring and procedures manual. Minneapolis, MN: National Computer Systems, Inc.

Derogatis, L., \& Melisaratos, N. (1983). The Brief Symptom Inventory: An introductory report. Psychological Medicine, 13, 595-605.

Douglas, K., Ogloff, J., Nicholls, T., \& Grant, I. (1999). Assessing risk for violence among psychiatric patients: The HCR-20 Violence Risk Assessment Scheme and the Psychopathy Checklist: Screening Version. Journal of Consulting and Clinical Psychology, 67, 917-930.

Drake, R., Mercer-McFadden, C., Mueser, K., McHugo, G., \& Bond, G. (1998). Review of integrated mental health and substance abuse treatment for patients with dual disorders. Schizophrenia Bulletin, 24, 589-608.

Gardner, W., Lidz, C., Mulvey, E., \& Shaw, E (1996). A comparison of actuarial methods for identifying repetitively violent patients with mental illnesses. Law and Human Behavior, 20, 35-48.

Gendreau, P. (1996). The principles of effective intervention with offenders. In A. Harland (Ed.), Choosing correctional options that work (pp. 117-130). Thousand Oaks, CA: Sage.

Gendreau, P., Little, T., \& Goggin, C. (1996). A meta-analysis of the predictors of adult offender recidivism: What works! Criminology, 34, 575-607.

Goldman, H., Frank, R., \& McGuire, T. (1994). Mental health care. In E. Ginzberg (Ed.), Critical issues in U.S. health reform (pp. 73-92). Boulder, CO: Westview.

Hakeem, M. (1948). The validity of the Burgess Method of parole prediction. American Journal of Sociology, 53, 376386.

Hanson, R., \& Harris, A., (1998). Dynamic predictors of sexual recidivism. (User Report No. 1998-01). Ottawa: Department of the Solicitor General of Canada. (Available at http:/ /www.sgc.gc.ca)

Hanson, R., \& Harris, A. (2000). The Sex Offender Need Assessment Rating (SONAR): A method for measuring change in risk levels. (User Report No. 2000-01). Ottawa: Department of the Solicitor General of Canada. (Available at http://www.sgc.gc.ca)

Heilbrun, K. (1997). Prediction versus management models relevant to risk assessment: The importance of legal decision-making context. Law and Human Behavior, 21, 347-359.

Henry, W. (1996). Structural analysis of social behavior as a common metric for programmatic psychopathology and psychotherapy research. Journal of Consulting and Clinical Psychology, 64, 1263-1275.
Koriat, A., Goldsmith, M., \& Pansky, A. (2000). Toward a psychology of memory accuracy. Annual Review of Psychology, 51, 481-537.

Kraemer, H.C., Kazdin, A.E., Offord, D.R., \& Kessler, R.C. (1997). Coming to terms with the terms of risk. Archives of General Psychiatry, 54, 337-343

Lidz, C.W., Mulvey, E.P., \& Gardner, W. (1993). The accuracy of predictions of violence to others. Journal of the American Medical Association, 269, 1007-1011.

Martino, S., Carroll, K., O’Malley, S., \& Rounsaville, B. (2000). Motivational Interviewing with psychiatrically ill substance abusing patients. American Journal on Addictions, 9, 8891.

Miller, W., \& Rollnick, W. (Eds.) (1991). Motivational Interviewing: Preparing people for change. New York: Guilford.

Monahan, J., Steadman, H., Silver, E., Appelbaum, P., Robbins, P., Mulvey, E., Roth, L., Grisso, T., \& Banks, S. (2001). Rethinking violence risk assessment: Mental disorder and the law. New York: Oxford University Press.

Quinsey, V., Harris, G., Rice, M., \& Cormier, C. (1998). Violent offenders: Appraising and managing risk. Washington, DC: American Psychological Association.

Skeem, J., \& Mulvey, E. (2002). Monitoring the violence potential of mentally disordered offenders being treated in the community. In A. Buchanan (Ed.), Care of the mentally disordered offender in the community (pp. 111-142). New York: Oxford Press.

Steadman, H.J., Mulvey, E.P., Monahan, J., Robbins, P.C., Appelbaum, P.S., Grisso, T., Roth, L.H., \& Silver, E. (1998). Violence by people discharged from acute psychiatric inpatient facilities and by others in the same neighborhoods. Archives of General Psychiatry, 55, 393-401.

Strauss, M., \& Gelles, R. (1990). Physical violence in American families. Brunswick, NJ: Transaction.

Swanson, A., Pantalon, M., \& Cohen, K. (1999). Motivational Interviewing and treatment adherence among psychiatric and dually diagnosed patients. Journal of Nervous and Mental Disease, 187, 630-635.

Swanson, J., Swartz, M., Borum, R., Hiday, V., Wagner, H., \& Burns, B. (2000). Involuntary out-patient commitment and reduction of violent behaviour in persons with severe mental illness. British Journal of Psychiatry, 176, 324-331.

U. S. Department of Health and Human Services (1999). Mental health: A report of the Surgeon General. Rockville, MD: U.S. Department of Health and Human Services, Substance Abuse and Mental Health Services Administration, Center for Mental Health Services, National Institutes of Health, National Institute of Mental Health.

Weissman, E., Pettigrew, K., Sotsky, S., \& Regier, D. (2000). The cost of access to mental health services in managed care. Psychiatric Services, 51, 664-666. 